\title{
3-(Tetrazol-5-yl)-2-imino-coumarins Derivatives: Synthesis, Characterization, and Evaluation on Tumor Cell Lines
}

\author{
Ali Bouattour'1, Mehdi Fakhfakh1, Souhir Abid El-Gharbi'2, Majdi Abid², Ludovic Paquin ${ }^{3,4}$, \\ Rémy Le Guevel ${ }^{5}$, Thierry Charlier ${ }^{5}$, Houcine Ammar' ${ }^{1}$, Jean-Pierre Bazureau $3,4^{*}$ (1) \\ ${ }^{1}$ Laboratoire de Chimie Appliquée: Hétérocycles, Corps Gras et Polymères, Faculté des Sciences de Sfax, Université de Sfax, \\ Sfax, Tunisie \\ ${ }^{2}$ Chemistry Department, College of Science and Arts, Jouf University, Al Qurayyat, Al Jawf, KSA \\ ${ }^{3}$ Institut des Sciences Chimiques de Rennes, ISCR UMR CNRS 6226, Campus Beaulieu, Université de Rennes 1, Bât. 10A, \\ Rennes Cedex, France \\ ${ }^{4}$ S2Wave Platform, ScanMAT UMS 2001 CNRS, Campus Beaulieu, Université de Rennes 1, Bât. 10A, \\ Rennes Cedex, France \\ ${ }^{5}$ ImPACcell Platform, SFR Biosit Bât. 8, Campus Villejean, Rennes Cedex, France \\ Email: ${ }^{\rtimes}$ jean-pierre.bazureau@univ-rennes1.fr
}

How to cite this paper: Bouattour, A., Fakhfakh, M., El-Gharbi, S.A., Abid , M., Paquin, L., Le Guevel, R., Charlier, T., Ammar, H. and Bazureau, J.-P. (2021) 3-(Tetrazol-5-yl)-2-imino-coumarins Derivatives: Synthesis, Characterization, and Evaluation on Tumor Cell Lines. International Journal of Organic Chemistry, 11, 24-34. https://doi.org/10.4236/ijoc.2021.111003

Received: January 7, 2021

Accepted: March 6, 2021

Published: March 9, 2021

Copyright (C) 2021 by author(s) and Scientific Research Publishing Inc. This work is licensed under the Creative Commons Attribution International License (CC BY 4.0).

http://creativecommons.org/licenses/by/4.0/ (c) (i) Open Access

\begin{abstract}
The first report of new 3-(tetrazol-5-yl)-2-iminocoumarin derivatives is described. The title compounds were prepared in two steps and were obtained in good yields (55-93\%). They have been fully characterized by ${ }^{1} \mathrm{H},{ }^{13} \mathrm{C} N M R$, FTIR, UV-Visible and HRMS. They were tested for their antiproliferative activities against six representative human tumor cell lines (Huh 7-D12, Caco2, MDA-MB231, HCT 116, PC3 and NCI-H727) and HaCat keratinocytes. Among them, compound $5 \mathbf{e}$ was active on HCT $116\left(\mathrm{IC}_{50} 15 \mu \mathrm{M}\right)$.
\end{abstract}

\section{Keywords}

2-Iminocoumarin, Tetrazole, Fluorescence, Tumor Cell Lines, HCT 116

\section{Introduction}

Tetrazole [1] belongs to a family of compounds bearing the highest number of nitrogen atoms and surprisingly, they do not exist in nature. Tetrazole is an important scaffold because they are integrated in many compounds that have applications in numerous fields such as in medicine, biochemistry, and pharmacology [2] [3] [4]. It's noteworthy that in pharmacology, 5 -substituted- $1 H$-tetrazoles are bio-isosters of carboxylic acids because they presented comparable $\mathrm{pK}_{\mathrm{a}}$ 
(tetrazole 4.5 - 4.9 vs carboxylic acid 4.2 - 4.4), they have a similar size and a near molecular electrostatic potential [5]. They undergo very similar receptor-ligand interactions [6], exhibit a prolonged half-life time because they enhanced the metabolic stability [7] [8] and the membrane penetration [9]. Food Drug Administration (FDA) approved 23 drugs that contain $1 H$ - or $2 H$-tetrazole substituents [10]. Among them as examples (Figure 1): losartan as angiotensin II receptor [11], irbesartan as Angiotensin Receptor Blocker (ARB) for the treatment of hypertension [12], cilostazol for peripheral vascular disease [13] and cefazolin as antibiotic [14].

In our group and as part of our program aimed at developing new methods for the preparation of new building blocks or, for the synthesis of 2-iminocoumarins showing potential biological properties dedicated to protection of rat tissues from isoproterenol toxicity [15] or, for cancer [16] [17], we were motivated in this work respectively for: 1) to prepare a class of hybrid derivatives of 2-iminocoumarins bearing a tetrazole pharmacophore, 2) to evaluate their antiproliferative activities on tumor cell lines and iii) to study their UV/visible properties.

\section{Results and Discussion}

\subsection{Synthesis and Characterizations}

The synthetic route towards the preparation of the title compounds 5 is outlined below. In the first step (Scheme 1), the 3-cyano-2-iminocoumarins 3 were synthesized from an equimolecular mixture of various substituted 2-hydroxy benzaldehyde 1 (1a: 2-hydroxy-3-methoxybenzaldehyde, 1b: 4-diethylamino-2- hydroxybenzaldehyde, 1c: 2,4-dihydroxybenzaldehyde, 1d: 2-hydroxynaphthaldehyde, 1e: 2-hydroxybenzaldehyde, 1f: 2-hydroxy-4-methoxybnezaldehyde, 1g: 5-bromo-2-hydroxybenzaldehyde, 1h: 3-ethoxy-2-hydroxybenzaldehyde) and
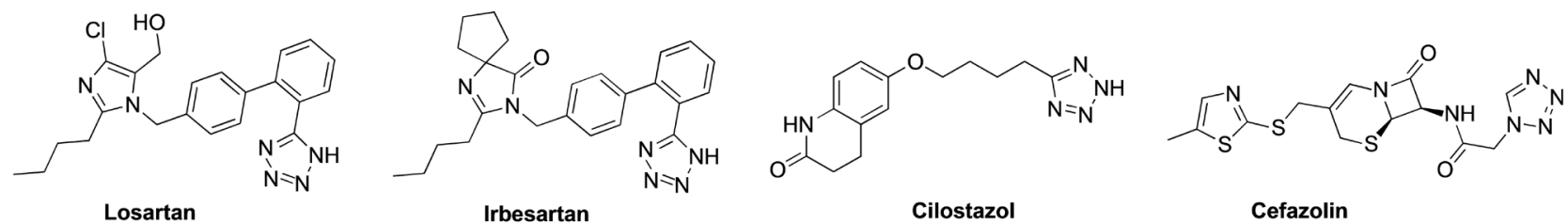

Figure 1. Selected drugs approved by FDA containing the tetrazole moiety.

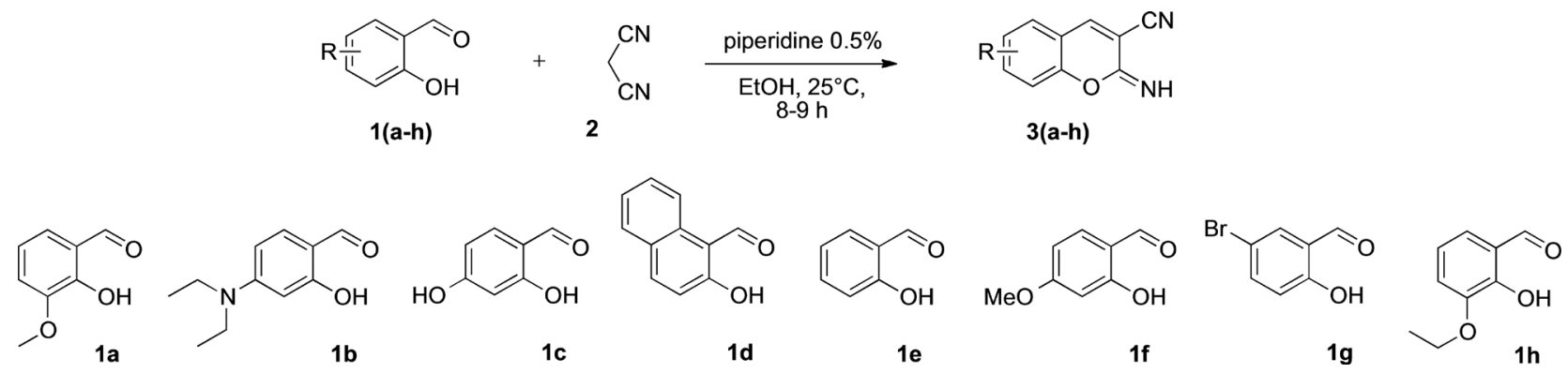

Scheme 1. Synthesis of 3-cyano-2-iminocoumarins 3(a-h) from various substituted 2-hydroxybenzaldehydes 1 (a-h) and propanedinitrile 2 . 
propanedinitrile 2 in ethanol with $0.5 \%$ of piperidine at room temperature. After a reaction time of 8 - 9 hours and elimination of volatile compounds in vacuo, the 3-cyano-2-iminocoumarins 3 were prepared easily according to this classical protocol previously developed in our laboratory [18] [19] [20].

In the second step (Scheme 2), transformation of the nitrile group of 3-cyano2-iminocoumarins 3 into desired tetrazole moiety [21] was accomplished by using one equivalent of zinc chloride as catalyst and sodium azide 4 . The reaction was conducted in a solution of THF and deionized water $(4: 1 \mathrm{v} / \mathrm{v})$ under reflux during 4 - $7 \mathrm{~h}$. Completion of the reaction was monitored by thin layer chromatography on 0.2-mm precoated plates of silica gel 60F-254 (Merck). After cooling down to room temperature, the precipitated material was collected by filtration on a Buchner funnel (porosity $\mathrm{N}^{\circ} 4$ ) and purified by washing with deionized water. The desired 3-(tetrazol-5-yl)-2-iminocoumarins 5 were synthesized in yields ranging from 55 to 92 (Table 1). Moderate yields must be mentioned for compounds $5 \mathrm{c}(61 \%)$ and $\mathbf{5 g}(55 \%)$ bearing respectively a phenolic function in C-7 position for $5 \mathrm{c}$ and a bromine atom in C-6 position for $5 \mathrm{~g}$. On the contrary, the presence of electron-donating groups, such as methoxy or ethoxy groups provide yields greater than $90 \%$, this concerns $5 \mathrm{a}$ (92\%) and $\mathbf{5 h}$ (93\%).<smiles>N#Cc1cc2cc[R]cc2oc1=N</smiles>
$3(a-h)$

$$
+\mathrm{NaN}_{3}
$$$$
4
$$<smiles>N=c1oc2cc[R1]cc2cc1-c1nnn[nH]1</smiles>

Scheme 2. Synthesis of substituted 3-(tetrazol-5-yl)-2-iminocoumarins 5(a-h) from various substituted 3-cyano-2-iminocoumarins $3(\mathrm{a}-\mathrm{h})$ and sodium azide 4 .

Table 1. Results for the preparation of 3-(tetrazol-5-yl)-2-iminocoumarins 5(a-h).

\begin{tabular}{|c|c|c|c|c|c|c|}
\hline & & & & & & \\
\hline Compound & $\mathrm{R}^{5}$ & $\mathrm{R}^{6}$ & $\mathbf{R}^{7}$ & $\mathbf{R}^{8}$ & Reaction time (h) & Yield (\%) \\
\hline $5 \mathrm{a}$ & $\mathrm{H}$ & $\mathrm{H}$ & $\mathrm{H}$ & $\mathrm{MeO}$ & 4 & 92 \\
\hline $5 b$ & $\mathrm{H}$ & $\mathrm{H}$ & $\mathrm{Et}_{2} \mathrm{~N}$ & $\mathrm{H}$ & 4 & 87 \\
\hline $5 c$ & $\mathrm{H}$ & $\mathrm{H}$ & $\mathrm{OH}$ & $\mathrm{H}$ & 7 & 61 \\
\hline $5 \mathrm{~d}$ & & & $\mathrm{H}$ & $\mathrm{H}$ & 4 & 73 \\
\hline $5 e$ & $\mathrm{H}$ & $\mathrm{H}$ & $\mathrm{H}$ & $\mathrm{H}$ & 4 & 72 \\
\hline $5 f$ & $\mathrm{H}$ & $\mathrm{H}$ & $\mathrm{MeO}$ & $\mathrm{H}$ & 6 & 84 \\
\hline $5 \mathrm{~g}$ & $\mathrm{H}$ & $\mathrm{Br}$ & $\mathrm{H}$ & $\mathrm{Br}$ & 6 & 55 \\
\hline $5 \mathrm{~h}$ & $\mathrm{H}$ & $\mathrm{H}$ & $\mathrm{H}$ & EtO & 6 & 93 \\
\hline
\end{tabular}

${ }^{a}$ Isolated yields. 
The structures for hybrid derivatives of 2-iminocoumarins $5(\mathrm{a}-\mathrm{h})$ were confirmed by ${ }^{1} \mathrm{H},{ }^{13} \mathrm{C}$ NMR, HRMS and FTIR. In the IR spectrum, the presence of $\mathrm{NH}$ stretching frequencies of the tetrazole group of 5 was detected at $3400 \mathrm{~cm}^{-1}$ [22] and associated to disappearance of the characteristic band at $2200-2300$ $\mathrm{cm}^{-1}$ for $\mathrm{CN}$ group of the 3-cyano-2-iminocoumarins 3 . This is also confirmed in ${ }^{13} \mathrm{C}$ NMR by the disappearance of a peak located at $\delta 115$ (attributed to the $\mathrm{CN}$ group of the starting compound 3) after synthesis of compounds 5. For MS, the $[\mathrm{M}+1]^{+}$molecular ion signal for all compounds $\mathbf{5}$ were obtained as base signal.

UV/Visible experiments were realized in DMSO and analytical data are reported in Table 2. Examination of these results shows that absorption maxima $\lambda_{\text {abs }}$ of compounds 5 are located between 320 to $444 \mathrm{~nm}$. Their emission peaks appears in purple-blue region due to a large Stokes shift (see Figure 2 for compound $\mathbf{5 b}$ in DMSO) and their fluorescence excitation spectra were similar. Highest values of quantum yields $\phi$ were observed respectively for compounds $\mathbf{5 c}(\phi=0.68)$ and moderate values for compounds $\mathbf{5 b}(\phi=0.362), 5 \mathrm{f}(\phi=0.335)$

Table 2. Maximum absorption $\left(\lambda_{\text {abs }}\right)$ and emission $\left(\lambda_{\text {em }}\right)$ wavelengths, and fluorescence quantum yields for the 3-(tetrazol-5-yl)-2-iminocoumarins $5(\mathrm{a}-\mathrm{h})$.

\begin{tabular}{cccc}
\hline Compound & $\lambda_{\text {abs }}(\mathrm{nm})$ & $\lambda_{\text {em }}(\mathrm{nm})$ & $\phi$ \\
\hline $5 \mathrm{a}$ & 328 & 449 & 0.042 \\
$5 \mathrm{~b}$ & 444 & 512 & 0.362 \\
$5 \mathrm{c}$ & 372 & 440 & 0.680 \\
$5 \mathrm{~d}$ & 392 & 460 & 0.208 \\
$5 \mathrm{e}$ & 340 & 414 & 0.034 \\
$5 \mathrm{f}$ & 366 & 439 & 0.335 \\
$5 \mathrm{~g}$ & 322 & 464 & 0.037 \\
$5 \mathrm{~h}$ & 320 & 454 & 0.081 \\
\hline
\end{tabular}

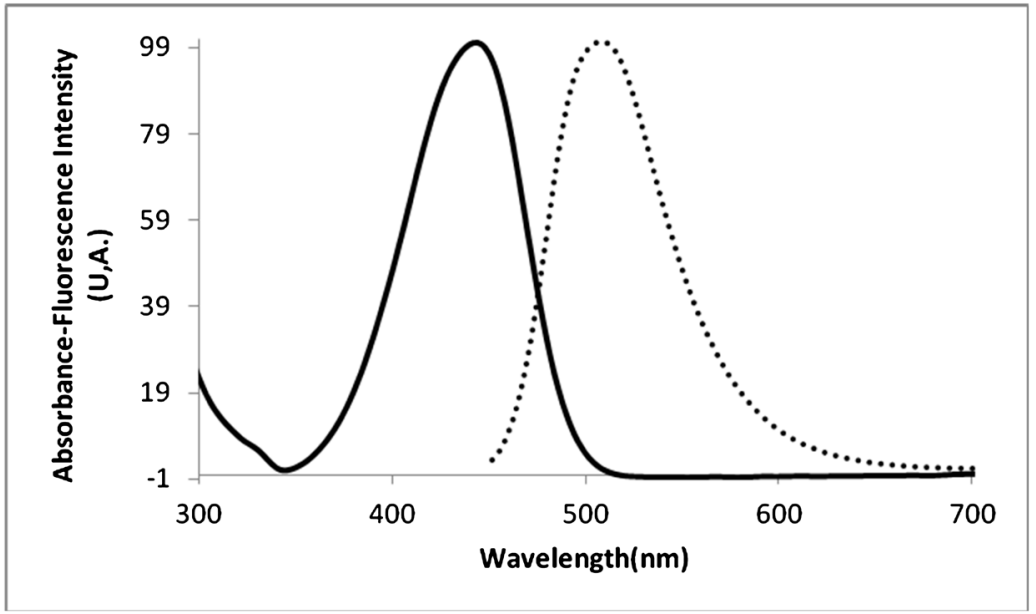

Figure 2. Normalized absorption (bold line) and emission (dotted line) spectrum of 2diethylamino-3-(tetrazol-5yl)-2-iminocoumarin 5b in DMSO. 
bearing a substituent in C-7 position. The presence of a donor group leaded to potential interesting quantum yields.

\subsection{Cytotoxic Assays}

The potential in vitro cytotoxic character of 3-(tetrazol-5-yl)-2-iminocoumarins $\mathbf{5}(\mathrm{a}-\mathrm{h})$ has been evaluated on six selected tumor cell lines, which are respectively Huh7-D12, Caco 2, MDA-MB231, HCT 116, PC3 and NCI-H727 and are representative of different cancers (leukemia, melanoma and cancers for liver, colon, breast, prostate, lung and kidney). HaCat keratinocyte was also used as normal cell line. For each tumor cell line, the $\%$ of cell survival was measured at a single dose of $25 \mu \mathrm{M}$ (after $48 \mathrm{~h}$ ) in triplicate. When the survival rate is less than $50 \%$, the $\mathrm{IC}_{50}$ value for this compound is determined, using roscovitine and doxorubicine as references for positive controls (Table 3 ). It can be observed that only compound $5 \mathbf{e}$ exhibited antiproliferative activity with a marked effect on HCT116 (5e: $\left.\mathrm{IC}_{50} 15 \mu \mathrm{M}\right)$.

\section{Conclusion}

This work described an easy method for the synthesis of new 3-(tetrazol-5-yl)-2imino-coumarins derivatives. Introduction of the tetrazol moiety on the 3-cyano function of 2 -iminocoumarins $\mathbf{3}(\mathrm{a}-\mathrm{h})$ involves a very simple method by using zinc chloride as catalyst that provided the desired compounds in yields ranging from $55 \%$ to $92 \%$. UV/Visible analytical data were also realized by measuring the absorption maxima $\lambda_{\mathrm{abs}}$, the emission maxima $\lambda_{\mathrm{em}}$ associated to fluorescence quantum yields $\phi$. Antiproliferative activities of all the new compounds were also

Table 3. Antiproliferative activities of 3-(tetrazol-5-yl)-2-iminocoumarins 5(a-h) on six tumor cell lines and HaCat keratinocyte.

\begin{tabular}{|c|c|c|c|c|c|c|c|}
\hline \multirow{2}{*}{ Compound } & \multicolumn{7}{|c|}{ Percentage of survival ${ }^{a}\left(\mathrm{IC}_{50}, \mu \mathrm{M}\right.$ of selected compounds) ${ }^{b}$} \\
\hline & Huh7-D12 & Caco 2 & MDA-MB231 & HCT 116 & PC3 & NCI-H727 & HaCat \\
\hline $5 a$ & 113 & 79 & 64 & 73 & 81 & 116 & 87 \\
\hline $5 b$ & 126 & 83 & 129 & 77 & 90 & 75 & 97 \\
\hline $5 c$ & 137 & 80 & 104 & 88 & 99 & 95 & 108 \\
\hline $5 d$ & 119 & 86 & 96 & 87 & 91 & 121 & 90 \\
\hline $5 e$ & $79(33)$ & $64(21)$ & $54(39)$ & $95(15)$ & $79(44)$ & $70(>25)$ & $92(38)$ \\
\hline $5 f$ & 118 & 81 & 124 & 93 & 123 & 124 & 102 \\
\hline $5 \mathrm{~g}$ & 143 & 92 & 127 & 110 & 98 & 95 & 114 \\
\hline $5 \mathrm{~h}$ & 226 & 86 & 96 & 95 & 133 & 198 & 85 \\
\hline Roscovitine & $21(15)$ & $3(15)$ & $21(12)$ & $10(9)$ & $24(13)$ & $30(43)$ & $6(11)$ \\
\hline Doxorubicine & $63(0.03)$ & $43(0.03)$ & $82(0.01)$ & $22(0.03)$ & 34 & 65 & $88(0.02)$ \\
\hline DMSO & $\begin{array}{c}100 \\
(>25)\end{array}$ & $\begin{array}{c}100 \\
(>25)\end{array}$ & $\begin{array}{c}100 \\
(>25)\end{array}$ & $\begin{array}{c}100 \\
(>25)\end{array}$ & $\begin{array}{c}100 \\
(>25)\end{array}$ & $\begin{array}{c}100 \\
(>25)\end{array}$ & $\begin{array}{c}100 \\
(>25)\end{array}$ \\
\hline
\end{tabular}

aPercentage of survival measured at $25 \mu \mathrm{M}$ (after $48 \mathrm{~h}$ using a single dose, triplicate). ${ }^{b} \mathrm{IC}_{50}$ values in parentheses are expressed in $\mu \mathrm{M}$ and are the average of three assays, standard error $\pm 0.5 \mu \mathrm{M}$. 
examined on six representative human tumor cell lines and we observed that compound $5 \mathbf{e}$ was active on HCT $116\left(\mathrm{IC}_{50} 15 \mu \mathrm{M}\right)$. These current results are the starting point of a new larger program within our group to investigate intensively the biological properties of these new compounds with potential applications in cancer.

\section{Experimental Sections}

\subsection{Chemistry}

\subsubsection{General Information}

Solvents were evaporated with a BUCHI rotary evaporator (New Castle, PA, USA). All reagents and solvents were purchased from Acros Fisher (Illkirch, France), Sigma-Aldrich Chimie (St Quentin Fallavier, France) and were used without further purification. ${ }^{1} \mathrm{H}$ NMR spectra were recorded on Bruker AC 300 $\mathrm{P}(300 \mathrm{MHz})$ spectrometer and ${ }^{13} \mathrm{C}$ NMR spectra on Bruker AC $300 \mathrm{P}(75 \mathrm{MHz})$ spectrometer (Bruker France Scientifique, Voisins-le-Bretonneux, France). Chemical shifts are expressed in parts per million downfield. Data are given in the following order: $\delta$ value, multiplicity ( $\mathrm{s}$, singlet; $\mathrm{d}$, doublet; $\mathrm{t}$, triplet; $\mathrm{q}$, quartet; quint: quintuplet, $\mathrm{m}$, multiplet; br, broad), number of protons, coupling constants $J$ is given in Hertz. The High-Resolution Mass Spectra (HRMS) were recorded in positive mode using direct Electrospray infusion, respectively on Waters Q-TOF 2 or on Thermo Fisher Scientific Q-Exactive spectrometers (Thermo Electron, Villebon-sur-Yvette, France) at the "Centre Régional de Mesures Physiques de l'Ouest” platform (CRMPO platform, ScanMAT UMS 2001 CNRS, Rennes, France). Melting points were determined on a Kofler melting point apparatus and were uncorrected. Infrared spectra were recorded on a Perkin Elmer 100 (Perkin Elmer France, Paris, France). UV/VIS absorption spectra were recorded on a Hewlett Packard 8452A diode array spectrophotometer (Hewlett Packard Enterprise, Paris, France). For solutions, corrected steady state fluorescence spectra were recorded with a Perkin Elmer LS55 spectrofluorometer using cells of $1 \mathrm{~cm}$ optical pathway (Perkin Elmer France, Paris, France). Elemental microanalyses were performed on an EA1112 analyzer from CE Instruments $\mathrm{Hi}$ Tech Detection Systems HTDS, ZI Charguia II 2035, Tunis-Carthage, Tunisie).

\subsubsection{General Procedure for the Synthesis of \\ 3-(tetrazol-5-yl)-2-iminocoumarins 5(a-h)}

To a stirred solution of 2-iminocoumarin-3-carbonitrile $3(0.27 \mathrm{mmol})$ in a solution of $4 \mathrm{~mL}$ of THF and $1 \mathrm{~mL}$ of deionized water, was added successively zinc chloride ( $37 \mathrm{mg}, 0.32 \mathrm{mml}$ ) and sodium azide $(20 \mathrm{mg}, 0.32 \mathrm{mmol})$. The resulting mixture was refluxed under magnetic stirring ( $500 \mathrm{rpm}$ ) for a reaction time from 4 to $7 \mathrm{~h}$. After cooling down to room temperature, the desired compound 5 was collected by filtration on a Büchner funnel (porosity $\mathrm{N}^{\circ} 4$ ), washed with cooled deionized water $(2 \times 2 \mathrm{~mL})$, dried under vacuum $(10-2 \mathrm{Torr})$ at $25^{\circ} \mathrm{C}$ for $1 \mathrm{~h}$ to give a powder.

1) 8-Methoxy-3-(tetrazol-5-yl)-2-iminocoumarin (5a) 
Yield $=92 \%$. Reaction time $=4 \mathrm{~h} . \mathrm{Mp}>260^{\circ} \mathrm{C} . \mathrm{IR}\left(\mathrm{cm}^{-1}\right): v=3295(\mathrm{NH})$, $1634(\mathrm{C}=\mathrm{N}) ;{ }^{1} \mathrm{H}$ NMR $\left(300 \mathrm{MHz}, \mathrm{CDCl}_{3}\right) \delta: 11.21$ (br s, $\left.1 \mathrm{H}, \mathrm{NH}\right), 9.32(\mathrm{~s}, 1 \mathrm{H}$, $\left.\mathrm{H}_{4}\right), 7.54\left(\mathrm{~m}, 3 \mathrm{H}, \mathrm{H}_{\mathrm{Ar}}\right), 4.02\left(\mathrm{~s}, 3 \mathrm{H}, \mathrm{OCH}_{3}\right) ;{ }^{13} \mathrm{C} \mathrm{NMR}\left(75 \mathrm{MHz}, \mathrm{CDCl}_{3}\right) \delta: 147.5$, $121.3,120.9,120.1,119.2,118,3$ 116.3, 112.5, 108.7, 56.6. Anal. Calcd for $\mathrm{C}_{11} \mathrm{H}_{9} \mathrm{~N}_{5} \mathrm{O}_{2}$ : C, 54.32; H, 3.73; N, 28.79. Found C, 54.26; H, 3.66; N, 28.71.

2) 7-N,N-Diethylamino-3-(tetrazol-5-yl)-2-iminocoumarin ( 5 b $)$

Yield $=87 \%$. Reaction time $=4 \mathrm{~h} . \mathrm{Mp}>260^{\circ} \mathrm{C}$. IR $\left(\mathrm{cm}^{-1}\right): v=3229(\mathrm{NH})$, $1643(\mathrm{C}=\mathrm{N}) ;{ }^{1} \mathrm{H}$ NMR $\left(300 \mathrm{MHz}, \mathrm{DMSO}-d_{6}\right) \delta: 11.20(\mathrm{br} \mathrm{s}, 1 \mathrm{H}, \mathrm{NH}), 8.81(\mathrm{~s}, 1 \mathrm{H}$, $\left.\mathrm{H}_{4}\right), 7.69\left(\mathrm{~d},{ }^{3} J=8.7 \mathrm{~Hz}, 1 \mathrm{H}, \mathrm{H}_{5}\right), 6.82\left(\mathrm{~d},{ }^{3} J=8.7 \mathrm{~Hz}, 1 \mathrm{H}, \mathrm{H}_{6}\right), 6.56\left(\mathrm{~s}, 1 \mathrm{H}, \mathrm{H}_{8}\right)$, $3.50\left(\mathrm{q},{ }^{3} \mathrm{~J}=13.8 \mathrm{~Hz}, 2 \mathrm{H}, \mathrm{CH}_{2}\right), 1.17\left(\mathrm{t},{ }^{3} \mathrm{~J}=13.8 \mathrm{~Hz}, 3 \mathrm{H}, \mathrm{CH}_{3}\right) ;{ }^{13} \mathrm{C} \mathrm{NMR}(75$ $\left.\mathrm{MHz}, \mathrm{DMSO}-d_{6}\right) \delta: 161.9,155.8,154.5,151.4,143.05,130.3,110.1,107.9,106.7$, 95.61, 44.2, 12.2. Anal. Calcd for $\mathrm{C}_{14} \mathrm{H}_{16} \mathrm{~N}_{6} \mathrm{O}: \mathrm{C}, 59.14 ; \mathrm{H}, 5.67 ; \mathrm{N}, 29.56$. Found C, 59.23; H, 5.61; N, 29.59 .

3) 7-Hydroxy-3 - (tetrazol-5-yl) -2-iminocoumarin (5c)

Yield $=61 \%$. Reaction time $=7 \mathrm{~h} . \mathrm{Mp}>260^{\circ} \mathrm{C}$. IR $\left(\mathrm{cm}^{-1}\right): v=3321(\mathrm{NH})$, $1646(\mathrm{C}=\mathrm{N}) ;{ }^{1} \mathrm{H}$ NMR $\left(300 \mathrm{MHz}, \mathrm{DMSO}-d_{6}\right) \delta: 8.97(\mathrm{br} \mathrm{s}, 1 \mathrm{H}, \mathrm{NH}), 8.89(\mathrm{~s}, 1 \mathrm{H}$, $\left.\mathrm{H}_{4}\right), 7.78\left(\mathrm{~d},{ }^{3} J=8.1 \mathrm{~Hz}, 1 \mathrm{H}, \mathrm{H}_{5}\right), 6.88\left(\mathrm{~d},{ }^{3} J=8.4 \mathrm{~Hz}, 1 \mathrm{H}, \mathrm{H}_{6}\right), 6.80\left(\mathrm{~s}, 1 \mathrm{H}, \mathrm{H}_{8}\right)$; ${ }^{13} \mathrm{C}$ NMR $\left(75 \mathrm{MHz}\right.$, DMSO- $\left.d_{6}\right) \delta: 163.1,160.6,155.7,153.8,139.4,130.9,114.4$, 111.0, 110.8, 101.7. Anal. Calcd for $\mathrm{C}_{10} \mathrm{H}_{7} \mathrm{~N}_{5} \mathrm{O}_{2}$ : C, 52.40; H, 3.08; N, 30.56 . Found C, 52.35; H, 3.05; N, 30.22.

4) 2-(Tetrazol-5-yI)-3-imino-3H-naphtho[2,1-b] pyran (5d)

Yield $=73 \%$. Reaction time $=4 \mathrm{~h} . \mathrm{Mp}>260^{\circ} \mathrm{C}$. IR $\left(\mathrm{cm}^{-1}\right): v=3262(\mathrm{NH})$, $1636(\mathrm{C}=\mathrm{N}) ;{ }^{1} \mathrm{H}$ NMR $\left(300 \mathrm{MHz}, \mathrm{DMSO}-d_{6}\right) \delta$ : $9.51(\mathrm{br} \mathrm{s}, 1 \mathrm{H}, \mathrm{NH}), 8.91(\mathrm{~s}, 1 \mathrm{H}$, $\left.\mathrm{H}_{4}\right), 8.72\left(\mathrm{~d},{ }^{3} J=9.0 \mathrm{~Hz}, 1 \mathrm{H}, \mathrm{H}_{5^{\prime} / 6^{6}}\right), 8.36\left(\mathrm{~d},{ }^{3} J=9.0 \mathrm{~Hz}, 1 \mathrm{H}, \mathrm{H}_{7}\right), 8.13\left(\mathrm{~d},{ }^{3} J=9.0\right.$ $\left.\mathrm{Hz}, 1 \mathrm{H}, \mathrm{H}_{5^{\prime} / 6^{\prime}}\right), 7.81\left(\mathrm{t},{ }^{3} J=6.1 \mathrm{~Hz}, 1 \mathrm{H}, \mathrm{H}_{5^{\prime \prime} / 6^{\prime \prime}}\right), 7.72\left(\mathrm{t},{ }^{3} \mathrm{~J}=3.0 \mathrm{~Hz}, 1 \mathrm{H}, \mathrm{H}_{5^{\prime \prime} / 6^{\prime \prime}}\right) ; 6.76$ $\left(\mathrm{d},{ }^{3} J=9.0 \mathrm{~Hz}, 1 \mathrm{H}, \mathrm{H}_{8}\right),{ }^{13} \mathrm{C} \mathrm{NMR}\left(75 \mathrm{MHz}, \mathrm{DMSO}-d_{6}\right) \delta: 154.0,149.7,139.9$, 135.4, 129.9, 128.9, 126.5, 122.3, 120.9, 116.4, 113.3, 112.6, 111.2, 109.4. Anal. Calcd for $\mathrm{C}_{14} \mathrm{H}_{9} \mathrm{~N}_{5} \mathrm{O}: \mathrm{C}, 63.87 ; \mathrm{H}, 3.45 ; \mathrm{N}, 26.61$. Found $\mathrm{C}, 63.95 ; \mathrm{H}, 3.41 ; \mathrm{N}$, 26.54.

\section{5) 3-( Tetrazol-5-yl)-2-iminocoumarin (5e)}

Yield $=72 \%$. Reaction time $=4 \mathrm{~h} . \mathrm{Mp}>260^{\circ} \mathrm{C} . \mathrm{IR}\left(\mathrm{cm}^{-1}\right): v=3313(\mathrm{NH})$, $1641(\mathrm{C}=\mathrm{N}) ;{ }^{1} \mathrm{H}$ NMR $\left(300 \mathrm{MHz}, \mathrm{DMSO}-d_{6}\right) \delta: 8.55(\mathrm{br} \mathrm{s}, 1 \mathrm{H}, \mathrm{NH}), 8.27(\mathrm{~s}, 1 \mathrm{H}$, $\left.\mathrm{H}_{4}\right), 8.02\left(\mathrm{dd}, 1 \mathrm{H}, \mathrm{H}_{8}\right), 7.76\left(\mathrm{~m}, 1 \mathrm{H}, \mathrm{H}_{6}\right), 7.55\left(\mathrm{dd}, 1 \mathrm{H}, \mathrm{H}_{5}\right), 7.48\left(\mathrm{~m}, 1 \mathrm{H}, \mathrm{H}_{7}\right) ;{ }^{13} \mathrm{C}$ NMR $\left(75 \mathrm{MHz}\right.$, DMSO- $\left.d_{6}\right) \delta: 153.5,149.5,144.2,133.7,129.8,125.0,120.8$, 118.3, 112.3, 109.3. Anal. Calcd for $\mathrm{C}_{10} \mathrm{H}_{7} \mathrm{~N}_{5} \mathrm{O}: \mathrm{C}, 56.34 ; \mathrm{H}, 3.31 ; \mathrm{N}, 32.85$. Found C, 56.37; H, 3.29; N, 32.88 .

6) 7-Methoxy-3-(tetrazol-5-yl)-2-iminocoumarin (5f)

Yield $=84 \%$. Reaction time $=6 \mathrm{~h} . \mathrm{Mp}>260^{\circ} \mathrm{C} . \mathrm{IR}\left(\mathrm{cm}^{-1}\right): v=3311(\mathrm{NH})$, $1640(\mathrm{C}=\mathrm{N}) ;{ }^{1} \mathrm{H}$ NMR $\left(300 \mathrm{MHz}, \mathrm{DMSO}-d_{6}\right) \delta: 8.99(\mathrm{br} \mathrm{s}, 1 \mathrm{H}, \mathrm{NH}), 8.52(\mathrm{~s}, 1 \mathrm{H}$, $\left.\mathrm{H}_{4}\right), 7.93\left(\mathrm{~d},{ }^{3} J=9.0 \mathrm{~Hz}, 1 \mathrm{H}, \mathrm{H} 5\right), 7.05\left(\mathrm{~d},{ }^{3} J=6.0 \mathrm{~Hz}, 1 \mathrm{H}, \mathrm{H}_{6}\right), 6.99\left(\mathrm{~s}, 1 \mathrm{H}, \mathrm{H}_{8}\right)$, $3.92\left(\mathrm{~s}, 1 \mathrm{H}, \mathrm{OCH}_{3}\right) ;{ }^{13} \mathrm{C} \mathrm{NMR}\left(75 \mathrm{MHz}\right.$, DMSO-d $\left.d_{6}\right) \delta: 164.3,155.8,149.7,144.5$, $131.15,121.4,111.9,109.8,108.2,100.5,56.2$. Anal. Calcd for $\mathrm{C}_{11} \mathrm{H}_{9} \mathrm{~N}_{5} \mathrm{O}_{2}: \mathrm{C}$, 54.32; H, 3.73; N, 28.79. Found C, 54.41; H, 7.67; N, 28.82.

7) 6, 8-Dibromo-3-(tetrazol-5-yl)-2-iminocoumarin (5g) 
Yield $=55 \%$. Reaction time $=6 \mathrm{~h} . \mathrm{Mp}>260^{\circ} \mathrm{C} . \mathrm{IR}\left(\mathrm{cm}^{-1}\right): v=3205(\mathrm{NH})$, $1651(\mathrm{C}=\mathrm{N}) ;{ }^{1} \mathrm{H}$ NMR $\left(300 \mathrm{MHz}, \mathrm{DMSO}-d_{6}\right) \delta: 9.46(\mathrm{br} \mathrm{s}, 1 \mathrm{H}, \mathrm{NH}), 8.62(\mathrm{~s}, 1 \mathrm{H}$, $\left.\mathrm{H}_{4}\right), 8.22\left(\mathrm{~s}, 1 \mathrm{H}, \mathrm{H}_{7}\right), 8.09\left(\mathrm{~s}, 1 \mathrm{H}, \mathrm{H}_{5}\right) ;{ }^{13} \mathrm{C}$ NMR (75 MHz, DMSO- $\left.d_{6}\right) \delta: 160.4$, $150,146.4,136.3,135.3,130.3,129.6,120.9,115.9,107.6$. Anal. Calcd for $\mathrm{C}_{10} \mathrm{H}_{5} \mathrm{~N}_{5} \mathrm{OBr}_{2}$ : C, 32.34; H, 1.35; N, 18.87. Found C, 32.39; H, 1.30; N, 18.92 .

8) 8-Ethoxy-3-(tetrazol-5-yl)-2-iminocoumarin ( $\mathbf{h}$ )

Yield $=93 \%$. Reaction time $=6 \mathrm{~h} . \mathrm{Mp}>260^{\circ} \mathrm{C} . \mathrm{IR}\left(\mathrm{cm}^{-1}\right): v=3305(\mathrm{NH})$, $1636(\mathrm{C}=\mathrm{N}) ;{ }^{1} \mathrm{H}$ NMR $\left(300 \mathrm{MHz}, \mathrm{DMSO}-d_{6}\right) \delta: 8.85(\mathrm{br} \mathrm{s}, 1 \mathrm{H}, \mathrm{NH}), 8.23(\mathrm{~s}, 1 \mathrm{H}$, $\left.\mathrm{H}_{4}\right), 7.35-7.43\left(\mathrm{~m}, 3 \mathrm{H}, \mathrm{H}_{\mathrm{Ar}}\right), 4.23\left(\mathrm{q},{ }^{3} J=12.0 \mathrm{~Hz}, 2 \mathrm{H}, \mathrm{OCH}_{2}\right), 1.43\left(\mathrm{t},{ }^{3} J=12.0\right.$ $\left.\mathrm{Hz}, 3 \mathrm{H}, \mathrm{CH}_{3}\right) ;{ }^{13} \mathrm{C}$ NMR $\left(75 \mathrm{MHz}\right.$, DMSO-d $\left.d_{6}\right) \delta: 145.6,144.5,142.9,125.1,121.0$, 120.8, 119.0, 116.6, 112.4, 109.5, 64.4, 14.4. Anal. Calcd for $\mathrm{C}_{12} \mathrm{H}_{11} \mathrm{~N}_{5} \mathrm{O}_{2}: \mathrm{C}, 56.03$; H, 4.31; N, 27.22. Found C, 55.98; H, 4.29; N, 27.29.

\subsection{Cell Culture and Survival Assays}

Caco2 (differentiated colorectal adenocarcinoma, Ref ECACC: 86010202), Huh7D12 (differential hepatocellular carcinoma, Ref ECACC: 01042712), MDA-MB231 (breast carcinoma, Ref ECACC: 92020424), HCT-116 (actively proliferating colorectal adenocarcinoma, Ref ECACC: 91091005), PC3 (prostate carcinoma, Ref ECACC: 90112714), NCI-H727 (lung carcinoma, Ref ECACC: 94060303) cell lines were obtained from the ECACC collection and HaCaT (keratinocyte from Cell Lines Service, Eppelheim, Germany). Cells were grown according to ECACC recommendations [23]. The toxicity test of the compounds on these cells was as follows: $2 \times 10^{3}$ cells for HCT-116 cells or $4 \times 10^{3}$ for the other cells were seeded in 96 multi well plates in triplicate and left for $24 \mathrm{~h}$ for attachment, spreading and growing. Then, cells were exposed for $48 \mathrm{~h}$ to increasing concentrations of the compounds, ranging from 0.1 to $25 \mathrm{mM}$ in a final volume of 120 $\mathrm{mL}$ of culture medium. Cells were fixed in cooled ethanol-acetic acid solution (90:5 v/v), nuclei were stained with Hoechst 3342 (Sigma) and counted using automated imaging analysis (Cellomics Arrayscan VTI/HCS Reader, Thermo/ Scientific). The $\mathrm{IC}_{50}$ were graphically determined.

\section{Funding}

One of us (A.B.) wishes to thank the "Ministère de l'Enseignement Supérieur et de la Recherche de Tunisie" for the grant. Financial support of this program carried out under the French cancer Institute "Cancéropôle Grand Ouest" in network "Marin molecules, Metabolism and Cancer" contract, is gratefully acknowledged.

\section{Acknowledgments}

The authors are grateful to the assistance of the staff (N. Le Yondre, P. Jéhan, F. Lambert) of CRMPO analytical chemistry core facility for HRMS analysis (CRMPO platform ScanMAT UMS 2001 CNRS, Université de Rennes 1, Bat. 11A, Campus de Beaulieu, Rennes, France). 


\section{Supplementary Materials}

Supplementary materials can be found at:

https://www.researchgate.net/profile/Jean Bazureau.

\section{Conflicts of Interest}

The authors declare no conflicts of interest regarding the publication of this paper.

\section{References}

[1] Neochoritis, C.G., Zhao, T. and Dömling, A. (2019) Tetrazoles via Multicomponent Reactions. Chemical Reviews, 119, 1970-2042. https://doi.org/10.1021/acs.chemrev.8b00564

[2] Wei, C.-X., Bian, M. and Gong, G.-H. (2015) Tetrazolium Compounds: Synthesis and Applications in Medicine. Molecules, 20, 5528-5553. https://doi.org/10.3390/molecules20045528

[3] Frija, L.M.T., Ismael, A. and Cristiano, M.L.S. (2010) Photochemical Transformations of Tetrazole Derivatives: Applications in Organic Synthesis. Molecules, 15, 3757-3774. https://doi.org/10.3390/molecules15053757

[4] Myznikov, L.V., Hrabalek, A. and Koldobskii, G.I. (2007) Drugs in the Tetrazole Series. (Review). Chemistry of Heterocyclic Compounds, 43, 1-9. https://doi.org/10.1007/s10593-007-0001-5

[5] Matta, C.F., Arabi, A.A. and Weaver, D.F. (2010) The Bioisosteric Similarity of the Tetrazole and Carboxylate Anions: Clues from the Topologies of the Electrostatic Potential and of the Electron Density. European Journal of Medicinal Chemistry, 45, 1868-1872. https://doi.org/10.1016/j.ejmech.2010.01.025

[6] Herr, R.J. (2002) 5-substituted-1 $H$-tetrazoles as Carboxylic Acid Isosteres: Medicinal Chemistry and Synthetic Methods. Bioorganic \& Medicinal Chemistry, 10, 3379 3393. https://doi.org/10.1016/S0968-0896(02)00239-0

[7] Holland, G.F. and Pereira, J.N. (1967) Heterocyclic Tetrazoles, a New Class of Lipolysis Inhibitors. Journal of Medicinal Chemistry, 10, 149-154. https://doi.org/10.1021/jm00314a004

[8] Figdor, S.K. and von Wittenau, M.S. (1967) Metabolism of 5-(3-Pyridyl)tetrazole. Journal of Medicinal Chemistry, 10, 1158-1159. https://doi.org/10.1021/jm00318a038

[9] Kubo, K., Kohara, Y., Yoshimura, Y., Inada, Y., Shibouta, Y., Furukawa, Y., Kato, T., Nishikawa, K. and Naka, T. (1993) Nonpeptide Angiotensin II Receptor Antagonists. Synthesis and Biological Activity of Potential Prodrugs of Benzimidazole7-Carboxylic Acids. Journal of Medicinal Chemistry, 36, 2343-2349. https://doi.org/10.1021/jm00068a011

[10] Wishart, D.S., Knox, C., Guo, A.C., Shrivastava, S., Hassanali, M., Stothard, P., et al. (2006) Drug Bank: A Comprehensive Resource for in Silico Drug Discovery and Exploration. Nucleic Acids Research, 34, D668-D672. https://doi.org/10.1093/nar/gkj067

[11] Royea, J., Zhang, L., Tong, X.-K. and Hamel, E. (2017) Angiotensin IV Receptors Mediate the Cognitive and Cerebrovascular Benefits of Losartan in a Mouse Model of Alzheimer's Disease. Journal of Neuroscience, 37, 5562-5573. https://doi.org/10.1523/JNEUROSCI.0329-17.2017 
[12] Mimran, A., Ruilope, L., Kerwin, L., Nys, M., Owens, D., Kassler-Taub, K. and Osbakken, M. (1998) A Randomised, Double-Blind Comparison of the Angiotensin II Receptor Antagonist, Irbesartan, with the Full Dose Range of Enalapril for the Treatment of Mild-to-Moderate Hypertension. Journal of Human Hypertension, 12, 203 208. https://doi.org/10.1038/sj.jhh.1000591

[13] Toyoda, K., Uchiyama, S., Yamaguchi, T., Donald Easton, J., Kimura, K., Hoshino, H., et al. (2019) Dual Antiplatelet Therapy Using Cilostazol for Secondary Prevention in Patients with High-Risk Ischemic Stroke in Japan. Lancet Neurology, 18, 539-548. https://doi.org/10.1016/S1474-4422(19)30148-6

[14] Zyzynska-Granica, B., Trzaskowski, B., Dutkiewicz, M., Zegrocka-Stendel, O., Machcinska, M., Bocian, K., Kowalewska, M. and Koziak, K. (2020) The Anti-Inflammatory Potential of Cefazolin as Common Gamma Chain Cytokine Inhibitor. Scientific Reports, 10, Article No. 2886. https://doi.org/10.1038/s41598-020-59798-3

[15] Khdhiri, E., Mnafgui, K., Ghazouani, L., Feriani, A., Hajji, R., Bouzanna, W., et al. (2020) (E)-N'-(1-(3-oxo-3H-benzo[f]chromen-2- yl)ethylidene)benzohydrazide Protecting Rat Heart Tissues from Isoproterenol Toxicity: Evidence from in Vitro and in Vivo Tests. European Journal of Pharmacology, 881, 173137-173146. https://doi.org/10.1016/j.ejphar.2020.173137

[16] Bouattour, A., Fakhfakh, M., Abid, S., Paquin, L., Le Guével, R., Corlu, A., Ruchaud, S., Bach, S., Ammar, H., et al. (2017) Microwave Assisted a Practical Synthesis of 4-imino-3-phenyl-3,4-dihydro-1 $H$-chromeno[2,3- $d$ ]pyrimidine-2(5H)-thione Derivatives and Exploration of Their Biological Activities. Arkivoc, 4, 291-302. https://doi.org/10.24820/ark.5550190.p010.040

[17] Bouattour, A., Fakhfakh, M., Abid, S., Paquin, L., Ammar, H. and Bazureau, J-P. (2017) An Efficient and Concise Synthesis of N-3-Substituted 9-Methoxy-4H-[1]benzopyrano[2,3-d]pyrimidine-4 $(5 \mathrm{H})$-imines and Formamidine Derivatives from Methyl N-(3-Cyano-8-methoxy-4H-[1]-benzopyran- 2-yl)methanimidate. Synthesis, 49, 3768-3774. https://doi.org/10.1055/s-0036-1588825

[18] Ammar, H., Fery-Forgues, S. and El Gharbi, R. (2003) UV/VIS Absorption and Fluorescence Spectroscopic Study of Novel Symmetrical Biscoumarin Dyes. Dyes and Pigments, 57, 259-265. https://doi.org/10.1016/S0143-7208(03)00028-7

[19] Fakhfakh, M., Turki, H., Fery-Forgues, S. and El Gharbi, R. (2010) The Synthesis and Optical Properties of Novel Fluorescent Iminocoumarins and Bis-Iminocoumarins: Investigations in the Series of Urea Derivatives. Dyes and Pigments, 84, 108-113. https://doi.org/10.1016/j.dyepig.2009.07.003

[20] Dammak, L., Kammoun, M., Ammar, H. and Abid, S. (2017) Synthesis and Antibacterial Activity of 2-Amino Chromenes Arising Cyanoiminocoumarins and $\beta$ Naphthol. Organic Communications, 10, 32-39.

https://doi.org/10.25135/acg.oc.6.16.11.442

[21] Zhou, Y., Yao, C., Ni, R. and Yang, G. (2010) Amine Salt-Catalyzed Synthesis of 5-subtituted $1 H$-tetrazoles from Nitriles. Synthetic Communications, 40, 2624-2632. https://doi.org/10.1080/00397910903318583

[22] Billes, F., Endrédi, H. and Keresztury, G. (2000) Vibrational Spectroscopy of Triazoles and Tetrazole. Journal of Molecular Structure: THEOCHEM, 530, 183-200. https://doi.org/10.1016/S0166-1280(00)00340-7

[23] Nakabayashi, H., Taketssa, K., Miyano, K., Yamane, T. and Sato, J. (1982) Growth of Human Hepatoma Cell Lines with Differentiated Functions in Chemically Defined Medium. Cancer Research, 42, 3858-3863. 


\section{Abbreviations}

FTIR: Fourier Transformed Infra Red

HRMS: High Resolution Mass Spectrometry

NMR: Nuclear Magnetic Resonance

UV: Ultra Violet 